\title{
Einstellungen und Motivation bezogen auf kulturell-ethnisch heterogene Schülerinnen- und Schülergruppen und ihre Bedeutung für differenzielle Instruktion im Unterricht
}

\author{
Miriam M. Gebauer $(\mathbb{D} \cdot$ Nele McElvany
}

Online publiziert: 3. August 2020

(C) Der/die Autor(en) 2020

Zusammenfassung Der Beitrag widmet sich der Frage, inwieweit Aspekte der professionellen Kompetenz von Lehrkräften von Bedeutung für einen diversitätssensiblen Unterricht sind. Der Fokus liegt dabei auf Einstellungen und motivationaler Orientierung von Lehrkräften und untersucht die Bedeutsamkeit für einen differenziellen Unterricht im Kontext kulturell-ethnischer Diversität. Daten von $N=250$ Lehrkräften wurden anhand von Strukturgleichungsmodellen analysiert sowie geschlechtsspezifische Unterschiede anhand von Mehrgruppenstrukturgleichungsmodellen geprüft. Die Ergebnisse weisen darauf hin, dass Einstellungen und motivationale Orientierung von substanzieller Relevanz für eine diversitätssensible Unterrichtsgestaltung sind. Eine Prüfung geschlechtsspezifischer Differenzen für die gefundenen Zusammenhangsmuster zeigen nur geringe Unterschiede. Die Ergebnisse heben die Bedeutung der professionellen Kompetenz von Lehrkräften für diversitätssensiblen Unterricht hervor und werden hinsichtlich ihrer Implikation für Forschung und Praxis diskutiert.

Schlüsselwörter Diversitätssensibler Unterricht · Einstellungen · Lehrkräfte · Motivationale Orientierung

Dr. M. M. Gebauer $(\bowtie) \cdot$ Prof. Dr. N. McElvany Institut für Schulentwicklungsforschung, Technische Universität Dortmund, Vogelpothsweg, 78, 44227 Dortmund, Deutschland

E-Mail: miriam.gebauer@tu-dortmund.de

Prof. Dr. N. McElvany

E-Mail: nele.mcElvany@tu-dortmund.de 


\title{
Attitudes towards culturally-ethnically heterogeneous groups of students and its meaning for individualized differentiated instruction in classroom context
}

\begin{abstract}
The present paper deals with the question to what extent aspects of the professional competence of teachers are important for diversity-sensitive teaching. It focuses on teachers' attitudes and motivational orientation toward cultural-ethnic diversity and their impact on individualized differential teaching. Data of $N=250$ teachers are examined using structural equation models and gender-specific differences are examined using multi-group structural equation models. The results indicate that attitudes and motivational orientations of teachers are important for diversity-sensitive teaching. An examination of gender-specific differences for the correlation patterns found showed only minor differences. The results highlight the importance of professional competence of teachers for diversity-sensitive teaching and are discussed with regard to their implications for research and practice.
\end{abstract}

Keywords Attitudes · Diversity-sensitive teaching $\cdot$ Motivation $\cdot$ Teacher

\section{Einleitung und Vorstellung der Forschungsfrage}

Schülerinnen und Schüler unterscheiden sich hinsichtlich einer Vielzahl an lernund leistungsrelevanten Disparitätsmerkmalen (Tillmann 2008; Gomolla 2009). Die durch Migration und demografischen Wandel bedingte, kulturell-ethnische Vielfalt von Schülerinnen und Schülern in Klassenzimmern gehört zu ebendiesen lern- und leistungsrelevanten Merkmalen (Gogolin 2006). Um Unterricht qualitätsvoll und schülerorientiert zu gestalten, sollten unter anderem individuelle Differenzen, wie die kulturell-ethnischen Unterschiede von Schülerinnen und Schülern, im Unterricht durch die Lehrkräfte berücksichtigt werden (Klieme und Warwas 2011). Die Voraussetzungen über die Lehrkräfte verfügen müssen, um einen diversitätssensiblen Unterricht zu konzertieren, umfassen neben dem Professionswissen auch Einstellungen und motivationale Aspekte (Baumert und Kunter 2006), da Einstellungen und motivationale Orientierungen bedeutsam für Verhalten und absichtsvolle Handlungen sind (Ajzen 2001). Allgemeine auf das Unterrichten bezogene Einstellungen oder Einstellungen bezogen auf soziale Merkmale von Schülerinnen und Schülern haben sich als relevant für die Gestaltung von Unterricht erwiesen (Staub und Stern 2002; Voss et al. 2013; Kumar et al. 2015) und andere empirische Befunde weisen auf geschlechtsspezifische Unterschiede bei Einstellungen hin, die angehende Lehrkräfte in Bezug auf das Unterrichten kulturell-ethnisch heterogener Lerngruppen haben (Gebauer und McElvany 2017). Anknüpfend daran stellen sich die Forschungsfragen des vorliegenden Beitrags: Sind heterogenitätsspezifische unterrichtsbezogene Einstellungen und die motivationale Orientierung von Lehrkräften an Gemeinschaftsschulen bezogen auf das Unterrichten von kulturell-ethnisch heterogenen Schülerinnen- und Schülergruppen von Bedeutung für die differenzielle Gestaltung des Unterrichts? Und können geschlechtsspezifische Differenzen festgestellt werden? 


\section{Theoretischer Rahmen und Forschungsbefunde}

\subsection{Heterogenität von Schülerinnen und Schülern}

Es existieren eine Vielzahl an lern- und leistungsrelevanten Merkmalen, die von Relevanz für den schulischen Bildungserfolg von Schülerinnen und Schülern sind (Tillmann 2008; Gomolla 2009). Zu diesen lern- und leistungsrelevanten Merkmalen zählen unter anderem individuelle kognitive oder motivationale Voraussetzungen der Schülerinnen und Schüler, beispielsweise unterschiedliches Vorwissen oder unterschiedliche intellektuelle Voraussetzung, wie die Kapazität des Arbeitsgedächtnisses (Hasselhorn und Grube 2003; Schneider und Stefanek 2004). Darüber hinaus unterscheiden sich Schülerinnen und Schüler hinsichtlich ihrer sozialen Herkunft, die neben dem sozioökonomischen Status der Familie, auch die kulturell-ethnische Herkunft der Schülerinnen und Schüler umfasst (Watermann und Baumert 2006). Kulturell-ethnische Vielfalt von Schülerinnen und Schülern gründet zumeist in der Migrationsgeschichte einer Familie und prägt, zum Beispiel durch unterschiedliche religiöse Ansichten und Riten, Wertvorstellungen und Einstellungen sowie sprachliche Voraussetzungen, die Handlungsmuster und Verstehensprozesse jener Schülerinnen und Schüler (Phinney et al. 2006; Wiater 2012). Mit Blick auf aktuelle Statistiken zeigt sich, dass aufgrund der Migrationsbewegungen der letzten Dekaden eine kulturell-ethnischen Vielfalt in den Klassen und Schulen vorzufinden ist, die sich aus Gruppen unterschiedlicher Herkunftsländer und Migrationsgründen zusammensetzt (vgl. BAMF 2019; Statistisches Bundesamt 2019). Aktuelle Befunde heben hervor, dass rund $40 \%$ der Schülerinnen und Schüler in Nordrhein-Westfälischen Schulen der Sekundarstufe I und II einen Migrationshintergrund haben, und mehrheitlich an Schulformen zu finden sind, die nicht zum Abitur führen (Henschel et al. 2019). Die Schulen des Modellversuchs der Gemeinschaftsschulen in NRW, in denen Schulformen mit und ohne Abiturmöglichkeit zusammengeführt wurden, haben zwischen 20-50\% Schülerinnen und Schüler, die einen Migrationshintergrund haben (Holtappels und McElvany 2017). Diese kulturell-ethnische Vielfalt von Schülerinnen und Schüler muss im Rahmen eines inklusiven, diversitätssensiblen Unterrichts durch die Lehrkräfte berücksichtigt werden. Zum einen werden demokratische Werte und Normen und die Wertschätzung einer pluralistischen Gesellschaft vermittelt (Scheunpflug und Affolderbach 2019). Zum anderen muss die Gestaltung individueller Lernprozesse orientiert an den Perspektiven, Wertvorstellungen und Normen der Schülerinnen und Schüler ausgerichtet und mit dem Unterrichtsgegenstand verknüpft werden. Dabei geht es insbesondere darum den Unterricht differenziell zu gestalten, orientiert an den kognitiven, konativen und sozialen Voraussetzungen der Schülerinnen und Schüler (Karakaşoğlu 2012; Gay 2013; Ladson-Billings 2014).

\subsection{Adaptiver Unterricht durch differenzielle Instruktion im Kontext kulturell- ethnischer Heterogenität von Schülerinnen und Schülern}

Unterricht ist der zentrale schulische Kontext in dem Wissenserwerbprozesse von Schülerinnen und Schülern durch Lehrkräfte initiiert, gesteuert und begleitet werden (Lipowsky 2006). Tiefenstrukturelle Qualitätsmerkmale von Unterricht, die von 
Bedeutung für die Lern- und Leistungsentwicklungen von Schülerinnen und Schülern sind, umfassen die kognitive Aktivierung, konstruktive Unterstützung und effiziente Klassenführung (Kunter und Voss 2011). Darüber hinaus wird als wichtig erachtet, den Unterricht orientiert an den individuellen Voraussetzungen und sozialen Bedingungen, die Schülerinnen und Schüler mitbringen, vorzubereiten, zu gestalten und nachzubereiten (Kyriakides et al. 2009; Klieme und Warwas 2011; Karakaşoğlu 2012). Ein Unterrichtskonzept zur individuellen Förderung von Schülerinnen und Schülern, das der Form des adaptiven Unterrichts zugeordnet werden kann, ist die differentiated instruction (differenzielle Instruktion, Oaksford und Jones 2001; Hall 2002). Sie basiert auf der theory of aptitude treatment interaction (Cronbach und Snow 1977) und geht von einer Wechselwirkung zwischen Lerninhalten und -methoden und den Fähigkeiten sowie Voraussetzungen der Schülerinnen und Schüler aus (Snow 1989). Durch die gleichzeitige Berücksichtigung von kognitiven und konativen Merkmalen der Schülerinnen und Schüler können Lehrkräfte durch mikroadaptive Instruktion den Leistungsstand, die Interessen und die motivationale Orientierung berücksichtigen (Snow 1989). Darüber hinaus bietet die makroadaptive Instruktion (Snow 1989) die Möglichkeit, soziokulturelle Unterschiede einzubeziehen, wie etwa die positive und diversitätssensible Berücksichtigung von differenziellen kulturell-ethnisch bedingten sozialen Regeln im Unterrichtskontext (Tharp 1989; Cole 2006). Von zentraler Bedeutung ist dabei eine Verbindung zwischen dem Unterrichtsstoff und den Voraussetzungen der Schülerinnen und Schüler herzustellen, indem auf die kulturellen Perspektiven jedes einzelnen oder jeder Gruppe von Schülerinnen und Schülern in einer schülerzentrierten, respektvollen und verständnisvollen Weise Bezug genommen wird (Tomlinson und Egan 2002; Au 2009). So werden beispielsweise in Halls (2002) learning cyclus model (Lernzyklus-Modell) zur Beschreibung differenzieller Instruktion die Voraussetzungen der Schülerinnen und Schüler als Ausgangslage betrachtet, aufgrund derer der Wissenserwerbsprozess und die Leistungsergebnisse der Schülerinnen und Schüler differieren. Voraussetzungen der Schülerinnen und Schüler sind dabei kognitive, konative und soziale Merkmale wie die kulturell-ethnische Herkunft der Familie. Schulische Lernprozesse werden als zyklisch beschrieben, die fortwährend durch die Lehrkräfte evaluiert und angepasst werden sollen (Hall 2002). Konstitutiv für eine gelingende Umsetzung eines differenziellen Unterrichts sind drei Phasen: die intensive Planung und Vorbereitung (Tricarico und Yendol-Hoppey 2012), die Durchführung der differenziellen Instruktion im Rahmen des Unterrichts (Levy 2008) und die Reflexion der differenziellen Instruktion im Nachgang des Unterrichts (Tomlinson 2014; Hall 2002). Dabei werden die beiden Komponenten intensive Vorbereitung und Reflexion, die zu möglichen Anpassungen und Überarbeitungen der differenziellen Instruktion führen, als Voraussetzung für eine gelungene und erfolgreiche Durchführung beschrieben (Tomlinson 2014). Empirische Evidenzen weisen darauf hin, dass Schülerinnen und Schüler von dieser Unterrichtsform hinsichtlich ihrer Leistungen, ihrer motivationalen Orientierung und ihres Wohlbefindens profitieren (z. B. Kyriakides et al. 2009; Tomlinson 2014). 


\subsection{Voraussetzungen und Bedingungen qualitätsvollen Unterrichts}

Modelle zur Beschreibung der professionellen Kompetenzen von Lehrkräften fassen die fachlichen und überfachlichen Voraussetzungen zusammen über die Lehrkräfte verfügen sollten, um einen qualitätsvollen und adaptiven Unterricht zu gestalten (Baumert und Kunter 2006). Neben dem Professionswissen, welches in das fachliche, fachdidaktische und erziehungswissenschaftliche Wissen unterschieden werden kann (Shulman 1986; Baumert und Kunter 2006; Blömeke et al. 2015), werden in diesen Modellen auch Überzeugungen, Werthaltungen, motivationale Orientierungen und selbstregulative Fähigkeiten genannt (Baumert und Kunter 2006). Fachliches, fachdidaktische und erziehungswissenschaftliches Wissen hat sich als effektiv für Leistungsmerkmale von Schülerinnen und Schülern erwiesen (z. B. Krauss et al. 2008) und ist bedeutsam für eine qualitätsvolle Unterrichtsgestaltung (z. B. Voss et al. 2014). Allgemeine lehr- und lernbezogene Überzeugungen darüber wie Wissensprozesse funktionieren, scheinen von Relevanz für die Gestaltung von Unterricht zu sein (Staub und Stern 2002). Auch sind auf die Lehrtätigkeit bezogene motivationale Orientierungen, wie beispielsweise die berufsbezogene Selbstwirksamkeitsüberzeugung von Lehrkräften, bedeutsam für eine qualitätsvolle Gestaltung von Unterricht oder die Leistungen von Schülerinnen und Schülern (z. B. Tschannen-Moran et al. 1998).

\subsection{Einstellungen und Motivation von Lehrkräften in Bezug auf das Unterrichten kulturell-ethnisch heterogener Schülerinnen- und Schülergruppen}

Es existiert eine Vielzahl an theoretischen Ausarbeitungen und Forschungsbefunden zu berufsbezogenen Vorstellungen, Überzeugungen oder Einstellungen von Lehrkräften (vgl. Reusser und Pauli 2014; Fischer et al. 2018). Diese oft synonym verwendeten Begriffe beschreiben das auf Erfahrungen basierende subjektive Wissen, welches sich aus kognitiven und affektiven Komponenten zusammensetzt (Pajares 1992; Calderhead 1996; Furinghetti und Pehkonen 2002; Hartinger et al. 2006). Eine Definition von Einstellungen liefern Eagly und Chaiken (1993), die diese als ,psychological tendency, expressed by evaluating a particular entity with some degree of favor or disfavor" (Eagly und Chaiken 1993, S. 1) bezeichnen (weitere Definitionen von Einstellungen siehe z. B. MODE Model Fazio 2007; oder APE Model Gawronski und Bodenhausen 2007). Einstellungen werden in kognitive, affektive und behaviorale Komponenten unterschieden (Maio und Haddock 2010). Kognitive, affektive oder behaviorale Assoziationen oder Erfahrungen addieren sich zu negierenden oder zustimmenden objektbezogenen Einstellungen, die sich im Weiteren in kognitiven, affektiven und behavioralen objektbezogenen Reaktionen zeigen (Eagly und Chaiken 1993). Dabei entspricht eine explizite, auf Wissen und Erfahrung basierende Objektbewertung, der kognitiven Einstellungskomponente (Eagly und Chaiken 1993; Maio und Haddock 2010). Dies können beispielsweise Bewertungen hinsichtlich der Nützlichkeit oder der aufzubringenden Kosten im Sinne eines zusätzlichen zeitlichen Arbeitsaufwandes sein (Ajzen 2001; Fazio 2007; Heckhausen und Heckhausen 2010; Bohner und Dickel 2011). Positive oder negative objektbezogene Emotionen werden der affektiven Einstellungskomponente zugeschrieben. Dazu 
gehören unter anderem negative Gefühle in Bezug auf negativ wahrgenommenes Stresserleben. Zur behavioralen Komponente gehören Handlungen und Verhaltensweisen aufgrund objektbezogener Einstellungen. Basierend auf Selbstwahrnehmungen vorangegangener einstellungsbezogener Handlungen (Bem 1972) entstehen oder festigen sich Einstellungen (Maio und Haddock 2010). In einer Reihe von Arbeiten, in denen die Begriffe Überzeugungen (beliefs) und Einstellungen (attitudes) synonym verwendet werden (z. B. Pajares 1992), werden Überzeugungen ebenfalls eine affektiv-motivationale Komponente zugeschrieben (Furinghetti und Pehkonen 2002; Hartinger et al. 2006; Fischer und Ehmke 2019). Berufsbezogene Überzeugungen werden dabei als normative Vorstellungen beschrieben, die Lehrkräfte in Bezug auf die Zusammensetzung und Generierung von Wissen (Epistemologische Überzeugungen) haben, oder in Bezug auf Schüler- oder Lehrkraftmerkmale oder bezogen auf kontextuelle Merkmale, wie Schule, Unterricht oder gesellschaftliche Bedingungen, wie beispielweise Überzeugungen in Bezug auf die Durchführung eines sprachsensiblen Unterrichts (Reusser und Pauli 2014; Fischer und Ehmke 2019). Ein belief oder Überzeugung in Bezug auf das Unterrichten, wäre dabei eine konkrete normative Vorstellung darüber, wie Unterricht sein sollte. Das kann zum Beispiel in transmissive und konstruktivistische Vorstellungen differenziert werden (Grigutsch et al. 1998; Dubberke et al. 2008; Biedermann et al. 2012), oder es umschreibt die Vorgehensweise zur Berücksichtigung sprachlicher Heterogenität im Unterricht (Fischer und Ehmke 2019). Im Gegensatz dazu gibt die gewählte theoretische, weniger normative Definition Auskunft darüber, inwieweit eine positive oder negative Bewertung der berufsbezogenen Tätigkeit vorliegt, demzufolge, ob das Unterrichten von kulturell-ethnisch heterogenen Schülergruppen als positiv im Sinne einer Nützlichkeit oder negativ, im Sinne von zusätzlichen Kosten eingeschätzt wird sowie, ob negative affektive Kosten wahrgenommen werden (Eagly und Chaiken 1993; Ajzen 2001; Dickel und Bohner 2012).

Neben Einstellungen ist die motivationale Orientierung ein zentraler verhaltensund handlungserklärender Faktor (Eccles und Wigfield 2002; Heckhausen und Heckhausen 2010). Die motivationale Orientierung setzt sich aus unterschiedlichen Facetten zusammen. Hierzu gehören unter anderem Zielorientierungen oder die wahrgenommenen selbstbezogenen Fähigkeitsüberzeugungen (vgl. z. B. Dweck und Elliott 1988; Bandura 1997). Eine weitere zentrale motivationale Facette ist die intrinsische Motivation, welches die Freude und Interesse am Lerngegenstand beschreibt, was dazu führt, dass eine Handlung um seiner selbst willen erfolgt (Deci und Ryan 1985). Darüber hinaus ist das Handlungsergebnis und die damit zusammenhängenden Erwartungen von Bedeutung für Handlung und Verhalten (Heckhausen 1989). Dementsprechend können die intrinsische Motivation und der erwartete Erfolg, neben den Einstellungen von Lehrkräften, als handlungserklärende Faktoren von Relevanz für die Gestaltung von Unterricht sein (Ajzen 2001; Eccles und Wigfield 2002). Differenzielle Untersuchungen zu Einstellungen von Lehrkräften in Bezug auf das Unterrichten heterogener Schülerinnen- und Schülergruppen zeigen, dass Einstellungen von Lehrkräften heterogenitätsspezifisch sind. Die Befunde deuten darauf hin, dass Lehrkräfte unterschiedliche Einstellungen in Bezug auf das Unterrichten sozial-, kulturell-ethnisch- und leistungsheterogener Schülergruppen haben (z.B. Merk et al. 2018). Darüber hinaus weisen Ergebnisse anderer Untersuchungen 
darauf hin, dass angehende Lehrkräfte bei positiveren Einstellungen in Bezug auf das Unterrichten kulturell-ethnisch heterogener Schülergruppen eine generelle Berücksichtigung von kulturell-ethnischer Heterogenität im Unterricht anstreben (Gebauer und McElvany 2017). Dabei sind in dieser Untersuchung die Intentionen und keine konkrete Unterrichtsform im Zusammenhang mit den Einstellungen untersucht worden.

\subsection{Determinierende Faktoren von Einstellungen und Motivation}

Differenzielle Befunde zwischen Lehrkräften lassen sich hinsichtlich des Merkmals Geschlecht sowie in Bezug auf Alter und Schulform feststellen (Huang 2007; Huang und Fraser 2009; Lam et al. 2010; Fischer und Ehmke 2019). So berichten weibliche Lehrkräfte positivere Einstellungen in Bezug auf kulturell-ethnische Vielfalt, höhere Motivation kulturell-ethnisch heterogene Lerngruppen zu unterrichten und sie sind eher dazu bereit kulturell-ethnische Heterogenität im Unterricht zu berücksichtigen (Columna et al. 2010; Ford und Quinn 2010; Hartwig et al. 2017; Fischer und Ehmke 2019). Darüber hinaus zeigen weibliche Lehrkräfte auch in Bezug auf andere Formen von Heterogenität eher die Bereitschaft diese im Unterricht zu berücksichtigen, als ihre männlichen Kollegen (Ellins und Porter 2005). Erklärungsansätze für geschlechtsspezifische Differenzen bei Einstellungen oder motivationalen Selbsteinschätzung verweisen auf geschlechterdifferenzielles Beantwortungsverhalten, wobei Frauen zu positiveren und sozial akzeptierten Antwortverhaltensweisen tendieren (Wigfield et al. 1996; Stocké 2004; Ellins und Porter 2005). Dabei wird davon ausgegangen, dass die eigene Geschlechtsrollenidentität, im Kontext von wahrgenommenen Geschlechtsrollenstereotypen und Anpassungsdruck diese zu erfüllen, in enger Wechselwirkung stehen und so zu geschlechtstypischen Verhalten führen (Trautner 1991; Hartmann und Trautner 2009). Ebenso führen geschlechtstypische Verhaltensweisen zu differenziellen kognitiven und affektiven Wahrnehmungen und Bewertungen des sozialen Umfelds (Trautner 1991; Ellemers 2018). So kommen normative Vorstellungen über sozial konstruierte weibliche oder männliche konnotierte Eigenschaften zum Tragen, die bedeutsam sind für soziale Interaktionen und Orientierung (Zemore et al. 2012).

Schulformdifferenzielle Unterschiede werden in Verbindung gebracht mit unterschiedlichen Ausbildungsgängen der jeweiligen Lehrämter und den spezifischen, den beruflichen Alltag determinierenden, Schulkontexten (z. B. Blömeke et al. 2010; Fives und Buehl 2012). Schulformdifferenzielle Unterschiede zwischen Lehrkräften in Bezug auf unterrichtsbezogene Einstellungen zeigten, dass angehende Lehrkräfte an Grund-Haupt und Realschulen eher dazu bereit sind die kulturell-ethnische Heterogenität von Schülerinnen und Schülern zu berücksichtigen (Schmotz und Blömeke 2009). Andere Forschergruppen berichteten, dass Grundschullehrkräfte positivere Bewertungen der sozialen Eingebundenheit und der Innovationbereitschaft berichten, als Lehrkräfte weiterführender Schulformen (Docker et al. 1989). Vergleichende Untersuchungen zu weiteren Aspekten der professionellen Kompetenz weisen auf schulformspezifische Unterschiede zwischen Lehrkräften in ihren fachspezifischen Kompetenzen hin (Brunner et al. 2006). Entsprechende Unterschiede können bereits am Ende der universitären Ausbildungsphase nachgewiesen werden. So können 
schulformspezifische Differenzen in fachübergreifenden Aspekten des professionellen Wissens bei Lehramtsstudierenden am Ende ihres Studiums festgestellt werden (König et al. 2008), wobei dies zu schulformdifferenzieller Unterrichtsgestaltung führen könnten (Korneck et al. 2017). Unterschiede zwischen Experten, Lehrkräften höheren Alters die infolgedessen über mehr Lehrerfahrungen verfügen, und Novizen, jüngere Lehrkräfte mit geringerer beruflicher Erfahrungen, lassen sich hinsichtlich einer Vielzahl an unterrichtsrelevanten Merkmalen beobachten (Berliner 2001; Fuller und Unwin 2004; Meschede et al. 2017). Dabei wird davon ausgegangen, dass ein reflektierter Umgang mit Erfahrungen bedeutsam ist für die unterrichtsbezogenen Überzeugungen von Lehrkräften sowie für das Unterrichten und die Lehrtätigkeit (Philipp 2007; Wyss 2013). Forschungsbefunden weisen auf darauf hin, dass Lehrkräfte mit mehr beruflicher Erfahrung, im Vergleich zu Lehrkräften mit weniger Lehrerfahrung, seltener auf geringfügige Unterrichtsstörungen eingehen, konstruktivistische lehr-lerntheoretische Überzeugungen zeigen, jedoch häufiger traditionelle Unterrichtsmethoden anwenden (Salvano-Pardieu et al. 2009; Saborit et al. 2016; Meschede et al. 2017).

\section{Fragestellungen und Hypothesen}

Die dargelegten theoretischen Ausführungen und die empirische Befundlage deuten darauf hin, dass Lehrkräfte unterrichtsbezogene heterogenitätsspezifische Einstellungen haben, das heißt, dass davon ausgegangen werden kann, dass sich Einstellungen in Bezug auf unterschiedliche Heterogenitätsformen differenzieren lassen (z. B. Merk et al. 2018). Weitere Untersuchungen weisen auf die Relevanz von Einstellungen bezogen auf das Unterrichten von kulturell-ethnisch heterogenen Schülergruppen für die Intention kulturell-ethnische Heterogenität im unterrichtlichen Kontext zu berücksichtigen hin (Gebauer und McElvany 2017). Darüber hinaus haben sich Einstellungen und motivationale Orientierung in anderen Studien als bedeutsam für die Unterrichtsqualität erwiesen (z. B. Staub und Stern 2002). Infolgedessen sollen Einstellungen und motivationale Orientierung von Lehrkräften, die diese in Bezug auf das Unterrichten kulturell-ethnisch heterogener Schülergruppen haben, im Zusammenhang mit dem Unterrichtskonzept der differenzierenden Instruktion untersucht werden. Mit der Unterrichtsform der differenzierenden Instruktion kann ein Unterrichtskonzept in den Blick genommen werden, das konzeptionell darauf ausgerichtet ist, die kognitiven, konativen und herkunftsbedingten Unterschiede von Schülerinnen und Schüler diversitätssensibel zu berücksichtigen (Hall 2002). Dabei sollen die Einstellungen über die Bewertung der Nützlichkeit, Kosten und negativen Emotionen sowie die motivationalen Orientierungen bei Lehrkräften an Gemeinschaftsschulen untersucht werden. Einstellungen in Bezug auf komplexe Tätigkeiten, wie das Unterrichten kulturell-ethnischer Schülergruppen, können dabei als explizite und bewusst wahrgenommene und reflektierte Bewertungen verstanden werden (Strack und Deutsch 2004). An Gemeinschaftsschulen sind Lehrkräfte aller Schulformen tätig, wie beispielsweise auch an Gesamtschulen oder in manchen Bundesländern an Stadtteilschulen, und die kulturell-ethnische Heterogenität der Schülerinnen und Schüler ist vergleichsweise höher, als an anderen Schulformen der Sekundarstufe I 
und II (vgl. Statistisches Landesamt NRW 2016; Holappels und McElvany 2017). Weitere empirische Befunde weisen darauf hin, dass Einstellungen geschlechtsspezifisch sind und, dass schulformspezifische Lehramtsbefähigungen und das Alter, als Indikator für eine längere berufliche Tätigkeit, von Bedeutung für die Unterrichtsgestaltung zu sein scheinen (z. B. Kooij et al. 2008; Salvano-Pardieu et al. 2009). Diese unterrichtsrelevanten Aspekte sollen bei der Untersuchung der Zusammenhänge zwischen Einstellungen und diversitätssensibler Unterrichtsgestaltung berücksichtigt werden.

Infolgedessen verfolgt der vorliegende Beitrag das Ziel, die Bedeutung von Einstellungen und Motivation von Lehrkräften für einen differenzierenden Unterricht zu untersuchen. Die Fragestellungen lauten wie folgt:

1. Sind Einstellungen und Motivation, die Lehrkräfte in Bezug auf das Unterrichten von kulturell-ethnisch heterogenen Klassen haben, von Bedeutung für differenzierenden Unterricht (Vorbereitung, Durchführung der Instruktion und Reflexion)?

H1 Es wird davon ausgegangen, dass Einstellungen und Motivation bedeutsam für die Vorbereitung, Durchführung und Nachbereitung von differenzierender Instruktion sind.

2. Sind zwischen männlichen und weiblichen Lehrkräften differenzielle Unterschiede der gefundenen Zusammenhänge feststellbar?

H2 Es wird vermutet, dass sich weibliche und männliche Lehrkräfte in ihren Einstellungs- und Motivationsausprägungen unterscheiden und diese bei weiblichen Lehrkräfte in einem höheren Maß bedeutsam für differenzierenden Unterricht ist.

3. Bleiben die gefundenen Zusammenhänge und Unterschiede unter Berücksichtigung weiterer Kovariaten (Alter und schulformspezifische Lehramtsbefähigung) konstant?

H3 Es wird davon ausgegangen, dass die Berücksichtigung weiterer Variablen keine Veränderungen der zuvor festgestellten Ergebnisse ergibt. Es wird darüber hinaus erwartet, dass das Alter der Lehrkräfte als Indikator für die Lehrerfahrung und die studierte Schulform von Bedeutung für eine diversitätssensible Unterrichtsgestaltung ist.

\section{Methode}

\subsection{Stichprobe}

Grundlage der Analysen für den vorliegenden Beitrag sind Daten von $N=250$ nordrhein-westfälischen Lehrkräften der Sekundarstufe I, die an Gemeinschaftsschulen tätig waren. Im Durchschnitt lag das Alter der im Rahmen der Studie befragten Lehrkräfte bei $M=39,32$ Jahren ( $S D=9,62$ Jahre), wobei dieser Wert unter dem 
durchschnittlichen Wert für Lehrkräfte in Nordrhein-Westfalen liegt (vgl. Statistisches Landesamt NRW 2016). Die Stichprobe beinhaltete Angaben von 68,4\% weiblichen Lehrkräften. Die Lehramtsbefähigung für die Primarstufe oder aller einzelnen Schularten der Sekundarstufe I haben $n=185$ Lehrkräfte und $n=65$ haben eine Lehramtsbefähigung für die Sekundarstufe II des Gymnasiums.

\subsection{Instrumente}

Differenzierende Instruktion Die drei Phasen Instruktionsvorbereitung, Instruktionsdurchführung und Instruktionsreflexion der differenzierenden Instruktion wurden mit jeweils einer Skala gemessen. Die Skala zur Messung der Instruktionsvorbereitung umfasste vier Items und wurde mittels eines sechsstufigen Antwortformats von $1=$ nie bis $6=$ sehr häufig erfasst. Ein Beispielitem lautete ,Ich verbringe Zeit damit, mir über die Anforderungen der Materialien und Methoden einer Unterrichtsstunde mit Blick auf unterschiedliche Schüler/innen Gedanken zu machen“. Die Skala zur Erfassung der Instruktionsdurchführung wurde anhand von neun Items mit einem sechsstufigen Antwortformat von $1=$ nie bis $6=$ in jeder Stunde gemessen. In diesem Fall lautete ein Beispielitem ,Ich achte darauf, dass alle Schülerinnen und Schüler den Unterrichtsstoff verstanden haben, bevor ich ein neues Thema beginne“. Die Skala zur Messung der Instruktionsreflexion umfasste fünf Items, die anhand eines vierstufigen Antwortformats von 1=trifft überhaupt nicht zu bis $4=$ trifft voll und ganz zu auszuwählen waren; ein Beispielitem hierzu lautete ,Ich reflektiere darüber, ob die Materialien und Methoden für unterschiedliche Schülergruppen adäquat waren“. Die Skalen wurden bereits in anderen Studien eingesetzt (Frey et al. 2009), in der die drei-Faktorenstruktur der Skalen gezeigt wurde (Hartwig und Schwabe 2018). Die Formulierungen der Items wurden für die Fragestellungen des vorliegenden Beitrages adaptiert. Mittelwerte, Standardabweichungen und Reliabilität der Skalen werden in Tab. 1 berichtet.

Einstellungen Es wurden drei Skalen zur Erfassung der Einstellungen eingesetzt. Die Nützlichkeit (,Die Schüler/innen profitieren durch Unterricht in kulturell-ethnisch heterogenen Lerngruppen hinsichtlich ihrer Persönlichkeitsentwicklung“), die Kosten (,Die kulturell-ethnische Heterogenität in unseren Schulklassen erfordert von den Lehrkräften einen zusätzlichen Arbeitsaufwand") und die negativen Emotionen ${ }^{1}$ (,Ich fühle mich angespannt bei dem Gedanken daran, die Kinder entsprechend ihrer kulturell-ethnischen Voraussetzungen zu unterrichten“) wurden jeweils anhand von fünf Items mit einem vierstufigen Antwortformat von 1= trifft überhaupt nicht zu bis $4=$ trifft voll und ganz zu erfasst. Die Skalen zur Erfassung der Einstellungen wurden bereits in einer Reihe von Studien, an diversen Stichproben eingesetzt (z.B.

\footnotetext{
1 Den theoretischen Beschreibungen von Eagly und Chaiken (1993) zufolge wird die kognitive von einer affektiven Einstellungskomponente unterschieden (vgl. auch Aijzen 2001; Fazio 2007). Dabei können kognitive Kosten in Bezug auf Arbeits- und Organisationsprozesse wahrgenommen werden, oder aber als psychologische Kosten im Sinne von negativen Emotionen (vgl. Flake et al. 2015). Eine ausführliche Diskussion zur validen Erfassung kognitiver und affektiver Kosten kann bei Flake et al. (2015) nachgelesen werden.
} 


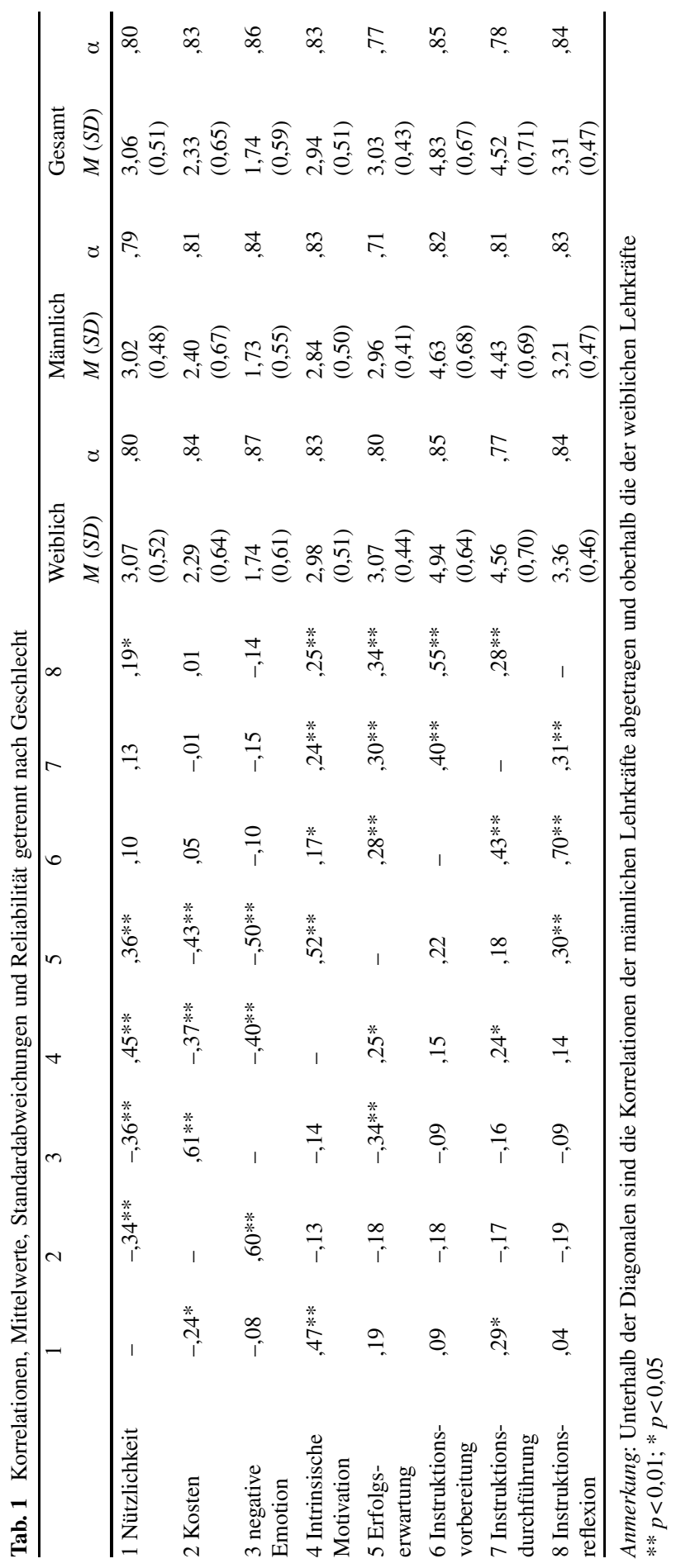


Merk et al. 2018). Mittelwerte, Standardabweichungen und Reliabilität der Skalen werden in Tab. 1 berichtet.

Motivation Es wurden zwei Skalen zur Erfassung der Motivation eingesetzt, die intrinsische Motivation und der erwartete Erfolg, jeweils bezogen auf das Unterrichten kulturell-ethnisch heterogener Schülergruppen. Die Skalen maßen die Motivationsfacetten anhand von jeweils fünf Items mit einem vierstufigen Antwortformat von $1=$ trifft überhaupt nicht zu bis $4=$ trifft voll und ganz zu. Die Skalen stammen aus der Skalenentwicklung der Studie Lehrer-H (vgl. Gebauer und McElvany 2013) und wurden bereits in anderen Studien eingesetzt (Hartwig et al. 2017; Merk et al. 2018). Ein Beispielitem für die intrinsische Motivation lautet wie folgt „Die kulturell-ethnische Heterogenität in unseren Schulklassen zu berücksichtigen, macht mir beim Unterrichten Spaß“. Ein Beispielitem für die Skala erwarteter Erfolg lautet „Ich bin ziemlich gut darin, den Unterricht entsprechend den kulturell-ethnischen Unterschieden meiner Schüler/innen zu gestalten“. Die Skalenmittelwerte, Standardabweichungen und Reliabilität werden in Tab. 1 illustriert.

Das Geschlecht der Lehrkräfte wurde im Rahmen der Fragebogenerhebung anhand der zwei prädominanten Geschlechtskategorien weiblich $=1$ und männlich $=2$ erfasst.

Weitere Kovariaten, die in die Analysen aufgenommen wurden, waren das $\mathrm{Al}$ ter als Indikator für die gesammelten Lehrerfahrungen, sowie das studierte Lehramt respektive Lehramtsbefähigung als Indikator für mögliche schulformspezifische Unterschiede, die auf die spezifischen Ausbildungsgänge zurückzuführen sind. Das Alter der Lehrkräfte wurde als metrische Werte in die Analysen aufgenommen, die studierte Schulform wurde als Gymnasium $=1$ und nicht Gymnasium $=0$ in den Analysen berücksichtigt.

\subsection{Analyseverfahren}

Die deskriptiven Auswertungen wurden mittels der Statistiksoftware IBM SPSS Statistics 22 durchgeführt. Es wurden Pearsons Korrelationskoeffizienten für bivariate Zusammenhänge zwischen den Skalen berichtet und einfaktorielle Varianzanalysen für die Prüfung der Mittelwertunterschiede zwischen weiblichen und männlichen Lehrkräften (MANOVA, Thorndike 1982) durchgeführt. Zur Beantwortung der Forschungsfragen eins und drei wurden Strukturgleichungsmodelle, und für die Forschungsfrage zwei Mehrgruppenstrukturgleichungsmodelle anhand der Statistiksoftware Mplus 8 (Byrne 2012; Asparouhov und Muthén 2014; Muthén und Muthén 2018) durchgeführt. Zur Einschätzung der Modelgüte werden die empfohlenen Gütekriterien comparative fit index (CFI), Tucker-Lewis index (TLI), root mean square error of approximation (RMSEA) und standardized root mean square residual (SRMR) berichtet (Hu und Bentler 1999).

Eine Prüfung der faktoriellen Struktur anhand von konfirmatorischen Faktorenanalysen (CFA) wurde für alle latenten Skalen durchgeführt, wobei jeweils ein Generalfaktormodell mit allen Skalen gegen ein dreifaktorielles Modell bei den Einstellungsskalen (Nützlichkeit, Kosten und negative Emotionen), respektive zweifaktorielles Modell für die Skalen zur Erfassung der motivationalen Orientierung (er- 
warteter Erfolg, intrinsische Motivation) getestet wurde. Die Ergebnisse der $\chi^{2}$ Differenztests zeigen für Einstellungsskalen für das dreifaktorielle Modell $\Delta \chi^{2}=152,97$, $\Delta d f=3, p<0,01$, und für die motivationalen Skalen für das Modell mit zwei Faktoren $\Delta \chi^{2}=59,19, \Delta d f=1, p<0,01$, eine bessere Modellanpassung.

Bei allen für die Analysen genutzten Skalen wurden weniger als $5 \%$ fehlende Werte festgestellt. Eine Ausnahme stellt die Skala erwarteter Erfolg dar, dort wurden für zwei Item 6,8 und $11,6 \%$ fehlende Werte beobachtet. Anhand des Tests von Little (1988) wurden die fehlenden Werte hinsichtlich ihrer Zufälligkeit überprüft, mit dem Ergebnis, dass für alle Skalen missing completely at random (MCAR, Rubin 1976) angenommen werden kann (Enders 2010). Demzufolge konnten die fehlenden Werte für die Strukturgleichungsmodelle, die mittels der Statistiksoftware Mplus durchgeführt wurden, anhand der Einstellung full information maximum likelihood (FIML) berücksichtigt werden (Rubin 1976; Little et al. 2013; Muthén und Muthén 2018).

Um die Abhängigkeit der Prädiktoren untereinander auszuschließen, die zu einer Unter- oder Überschätzung der Koeffizienten führen könnte, wurde eine Kollinearitätsprüfung durchgeführt (Schneider 2009). Kollinearität würde vorliegen, wenn ein Toleranzwert von 0,40 vorliegt bzw. ein Varianzinflationsfaktor (VIF) von 10,00 (Fickel 2001; Belsley et al. 2004). Die Prüfung ergab für die Abhängigkeit der Variablen dieser Untersuchung Toleranzwerte zwischen 0,70 bis 0,96 und VIF Werte zwischen 1,03 bis 1,77. Demnach liegt keine Kollinearität zwischen den Prädiktoren vor.

Die Intraklassenkorrelationen (ICC) zur Prüfung des Verhältnisses der Varianz zwischen den Clustern zur Gesamtvarianz lagen für alle Skalen zwischen 0,03 und 0,07, was auf eine geringe systematische Variation zwischen den Schulen schließen lässt. Die Mehrebenenstruktur der Lehrkräfte in unterschiedlichen Schulen wurde aufgrund der geringen ICC und der nicht ausreichenden Anzahl von Schulen $(N=12)$ in den Analysen nicht berücksichtigt (Maas und Hox 2005).

\section{Ergebnisse}

\subsection{Deskriptive Auswertungen}

Die deskriptiven Ergebnisse werden in Tab. 1 dargestellt. Die Mittelwerte werden global sowie für weibliche und männliche Lehrkräfte getrennt berichtet. Die Prüfung der Mittelwertunterschiede auf Signifikanz zeigt für die Skalen Instruktionsvorbereitung $\left(F=11,01 ; p<0,01 ; \eta^{2}=0,05\right)$, Instruktionsdurchführung $(F=3,85 ; p=0,05$; $\left.\eta^{2}=0,02\right)$, Instruktionsreflexion $\left(F=3,85 ; p=0,06 ; \eta^{2}=0,02\right)$ intrinsische Motivation $\left(F=2,88 ; p=0,09 ; \eta^{2}=0,01\right)$ und erwarteter Erfolg $\left(F=3,19 ; p=0,07 ; \eta^{2}=0,01\right)$ bedeutsame Differenzen, wobei bei allen Mittelwerten höhere Ausprägungen bei den weiblichen Lehrkräften festgestellt werden können. Die bivariaten Korrelationen zeigen für die weiblichen Lehrkräfte bedeutsame Zusammenhänge zwischen der Nützlichkeit und der Instruktionsreflexion. Zwischen der intrinsischen Motivation und dem erwarteten Erfolg können für alle drei Instruktionsskalen bedeutsame bivariate Zusammenhänge beobachtet werden. Bei den männlichen Lehrkräften sind 
signifikante bivariate Zusammenhänge zwischen der Nützlichkeit und der Instruktionsdurchführung zu beobachten. Die motivationalen Orientierungen sind bei den männlichen Lehrkräften für die Instruktionsdurchführung und die Instruktionsreflexion bedeutsam.

\subsection{Ergebnisse Forschungsfrage 1}

Die erste Forschungsfrage lautete: Sind Einstellungen und Motivation, die Lehrkräfte in Bezug auf das Unterrichten von kulturell-ethnisch heterogenen Klassen haben, von Bedeutung für differenzierenden Unterricht (Vorbereitung, Durchführung und Reflexion differenzierender Instruktion)?

Die Ergebnisse der ersten Forschungsfrage werden in Abb. 1 berichtet. Die Gütekriterien weisen auf eine gute bis akzeptable Anpassung des Modells an die Daten hin $(\mathrm{CFI}=$,95; TLI= 94; RMSEA = ,03; SRMR= ,06). Bedeutsame Zusammenhänge zeigen sich zwischen der Nützlichkeit, der Instruktionsdurchführung und der Instruktionsreflexion. Ebenfalls von Bedeutung sind die Zusammenhänge zwischen den Skalen intrinsische Motivation, Instruktionsdurchführung und Instruktionsreflexion sowie zwischen den Skalen erwarteter Erfolg, Instruktionsdurchführung und Instruktionsreflexion. Die beiden negativ konnotierten Skalen zur Erfassung der Kosten im Sinne eines zusätzlichen Arbeitsaufwandes oder der negativen emotionalen

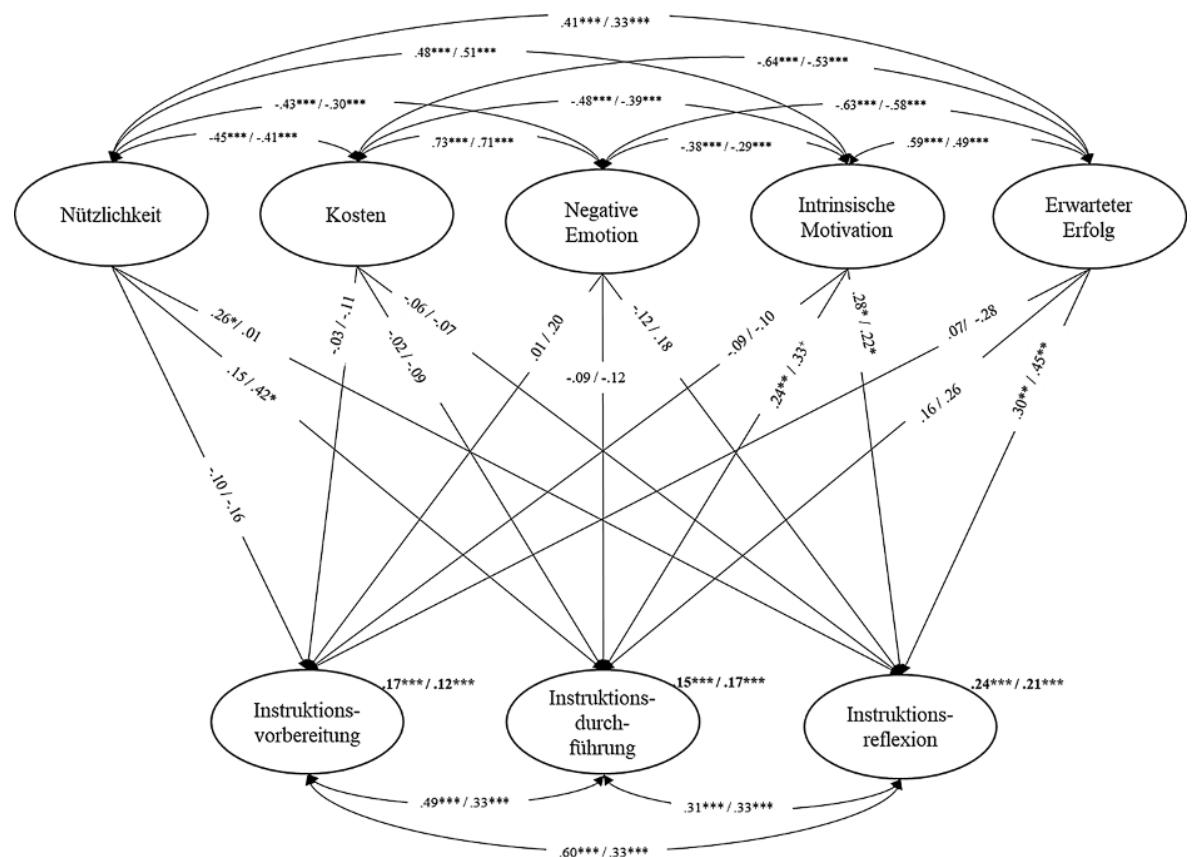

Abb. 1 Ergebnisse der Strukturgleichungsmodelle Forschungsfrage 1 und 3. Anmerkung: Die erstgenannten Koeffizienten berichten die Ergebnisse der ersten Forschungsfrage und die letztgenannten, die der dritten Forschungsfrage. ${ }^{+} p<0,1 .{ }^{*} p<0,05$. $* * p<0,01$. $* * * p<0,001$ 
Wahrnehmungen scheinen nicht von Relevanz für diese Form der differenzierenden Unterrichtsgestaltung zu sein.

\subsection{Forschungsfrage 2}

Die zweite Forschungsfrage lautete: Sind differenzielle Unterschiede der gefundenen Zusammenhänge zwischen männlichen und weiblichen Lehrkräften feststellbar?

Die Ergebnisse der differenziellen Analysen zur Beantwortung der zweiten Forschungsfrage werden in Abb. 2 illustriert. Die Gütekriterien deuten auf eine gute bis akzeptable Anpassung des Modells an die Daten hin (CFI=,94; TLI=,94; RMSEA=,04; SRMR =,06). Hinsichtlich der Zusammenhänge zwischen der Nützlichkeit, der Instruktionsdurchführung und der Instruktionsreflexion zeigt sich, dass die Nützlichkeit für weibliche Lehrkräfte von Bedeutung für die Instruktionsreflexion ist, nicht aber für männliche Lehrkräfte. Für männliche Lehrkräfte zeigen sich signifikante Pfadkoeffizienten zwischen der Nützlichkeit und der Instruktionsdurchführung, nicht aber für weibliche Lehrkräfte. Die globalen signifikanten Zusammenhänge zwischen der Skala intrinsische Motivation und den Skalen Instruktionsdurchführung und Instruktionsreflexion bleiben in der Tendenz sowohl für die weiblichen als auch für die männlichen Lehrkräfte bestehen. Für die Zusammenhänge zwischen

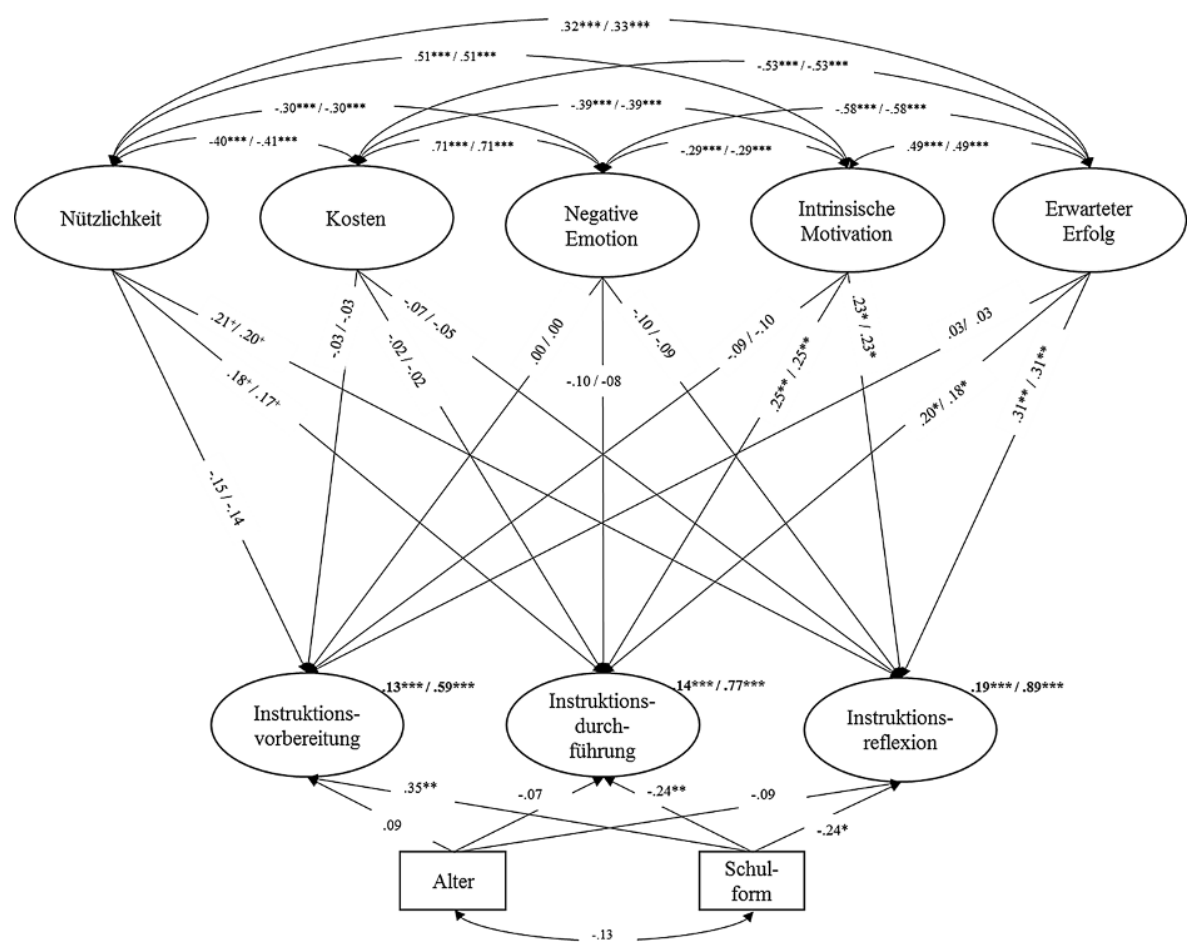

Abb. 2 Ergebnisse des Mehrgruppenstrukturgleichungsmodells Forschungsfrage 2. Anmerkung: Die erstgenannten Koeffizienten berichten die Ergebnisse der weiblichen Lehrkräfte und die letztgenannten, die der männlichen Lehrkräfte. ${ }^{+} p<0,1 .{ }^{*} p<0,05 .{ }^{*} p<0,01 . * * * p<0,001$ 
der Skala erwarteter Erfolg und den Instruktionsskalen bleibt bei der subgruppenspezifischen Betrachtung nur noch der Zusammenhang zwischen dem erwarteten Erfolg und der Instruktionsreflexion sowohl für weibliche als auch für männliche Lehrkräfte bestehen.

\subsection{Forschungsfrage 3}

Die dritte Forschungsfrage lautete: Bleiben die gefundenen Zusammenhänge und Unterschiede unter Berücksichtigung weiterer Kovariaten, konstant?

Die Ergebnisse der Analysen zur Prüfung der Fragstellung werden in Abb. 1 berichtet. Die Gütekriterien weisen auf eine gute bis akzeptable Anpassung des Modells an die Daten hin (CFI=,93; TLI=,92; RMSEA =,04; $\mathrm{SRMR}=, 07)$. Dabei kann für alle beobachteten Zusammenhänge, nach der Berücksichtigung des $A l$ ters und der Schulform, tendenziell konstant bleibende Pfadkoeffizienten festgestellt werden. Die Veränderungen vor und nach Kontrolle der Kovariaten überschreiten den Wert 2 der zweiten Nachkommastelle der Pfadkoeffizienten nicht. Die Zusammenhänge zwischen der Kontrollvariablen Alter und den Instruktionsvariablen sind nicht statistisch signifikant. Zwischen der Variable Schulform und den Unterrichtsvariablen sind für alle drei Skalen statistisch signifikante Unterschiede feststellbar. Dabei weisen die negativen Pfadkoeffizienten darauf hin, dass Lehrkräfte die eine Lehramtsbefähigung für die Primarstufe und Sekundarstufe I haben, eher zu einer differenzierenden Unterrichtsvorbereitung, -durchführung und -reflexion tendieren. Dahingegen sind positive statistisch signifikante Zusammenhänge zwischen der Variable Schulform und der Instruktionsvorbereitung festzustellen, die darauf hinweisen, dass Lehrkräfte mit einer Lehrbefähigung für die Schulform Gymnasium eher dazu tendieren die kulturell-ethnische Vielfalt in ihrer Unterrichtsvorbereitung zu berücksichtigen.

\section{Diskussion}

Ziel des vorliegenden Beitrags war die Prüfung der Bedeutung von unterrichtsbezogenen und kulturell-ethnisch differenziellen Einstellungen sowie motivationaler Orientierung von Lehrkräften für die Vorbereitung, Durchführung und Reflexion differenzierender Instruktion. Darüber hinaus sind geschlechtsspezifische Differenzen und geschlechterdifferenzielle Zusammenhänge geprüft worden, sowie weitere Kontrollvariablen berücksichtigt worden. Die Ergebnisse bestätigen die Bedeutung der Einstellungsskala Nützlichkeit für die differenzierende Instruktionsdurchführung und für die Instruktionsreflexion, die bereits in anderen Studien festgestellt werden konnte (z.B. Hartwig und Schwabe 2018). Wenn Lehrkräfte einer kulturell-ethnischen Diversität einen positiven Wert zu ordnen, richten Sie ihren Unterricht demzufolge an den Sichtweisen ihrer Schülerinnen und Schüler aus und bieten einen diversitätssensiblen Unterricht an (vgl. Kumar et al. 2015). Die negativ konnotierten Einstellungsskalen, Kosten im Sinne eines zusätzlichen Arbeits- und Organisationsaufwandes und negative Emotionen, als belastend wahrgenommene Stressempfindungen, sind nicht von Bedeutung für keine der drei Phasen der diffe- 
renziellen Instruktion. Diese Ergebnisse stehen ebenfalls im Einklang mit anderen Untersuchungsergebnissen (Hartwig und Schwabe 2018). Organisationsbezogenen und emotionale Kosten scheinen psychologisch messbare Konstrukte zu sein, die aber selten von Bedeutung für Verhalten oder Handlungen zu sein scheinen (vgl. dazu zusammenfassend Flake et al. 2015). Die motivationalen Orientierungen der Lehrkräfte sind, wie zu erwarten, bedeutsam für Durchführung und Reflexion eines diversitätssensiblen Unterrichts. Dabei sind die motivationalen Orientierungen relevant für die Performanz und diversitätssensible Unterrichtsgestaltung sowie für die anschließende Bewertung und mögliche Korrektur und Verbesserung der bisherigen Vorgehensweise. Forschungsbefunde anderer Studien fanden vergleichbare Ergebnisse wobei die motivationale Orientierung von Lehrkräften von Bedeutung für eine schülerorientierte Unterrichtsgestaltung ist (Bernaus et al. 2009; Klassen et al. 2011).

Die festgestellten geschlechtsspezifischen Unterschiede weisen darauf hin, dass weibliche Lehrkräfte höhere motivationale Ausprägungen haben und gleichzeitig eher dazu bereit sind diversitätssensiblen Unterricht anzubieten. Dies steht im Einklang mit Forschungsbefunden anderer Studien die fanden, dass weibliche Lehrkräfte eher dazu tendieren Unterricht nicht-traditionell und schülerorientiert zu gestalten (z. B. Bress 2000; Barber 2002; Starbuck 2003; Evans 2004; Gebauer und McElvany 2017).

Hinsichtlich der geschlechterdifferenziellen Zusammenhänge konnten für weibliche, als auch männliche Lehrkräfte statistisch signifikante Zusammenhänge zwischen der Nützlichkeit und den Instruktionsvariablen festzustellen, jedoch ist die Nützlichkeit des Unterrichtens kulturell-ethnischer Lerngruppen bei männlichen Lehrkräften für die Instruktionsdurchführung und bei weiblichen Lehrkräften für die Instruktionsreflexion relevant. Dabei scheint die Einschätzung der Nützlichkeit für weibliche Lehrkräfte für die Evaluation und mögliche Neuausrichtung des zukünftigen Unterrichts bedeutsam zu sein, wohingegen männliche Lehrkräfte von der Einschätzung der Nützlichkeit hinsichtlich der Instruktionsdurchführung, also der Performanz profitieren. Übliche Erklärungen für geschlechtsspezifische Unterschiede, wie beispielsweise, dass weibliche Studienteilnehmerinnen zumeist positivere und sozial erwünschte Aussagen treffen und dies auf eigene geschlechtsspezifische Stereotype und Geschlechtsrollenorientierung zurückzuführen ist (Eccles 2011; Hannover und Kessels 2011), liefern keine schlüssigen Begründungen für diese differenziellen Zusammenhänge. Vielmehr könnte dieser Unterschied in einer möglichen geschlechterdifferenziellen Verfahrensweise der Unterrichtsgestaltung - und reflexion liegen. Infolgedessen wäre es möglich, dass die Nützlichkeit von differenzieller Bedeutung für diese Unterrichtsphase ist, da diese Unterrichtsphase für weibliche respektive männliche Lehrkräfte bedeutsamer ist.

Die differenzielle Bedeutung der schulformspezifischen Lehramtsbefähigung deutet darauf hin, dass Lehrkräfte unterschiedlicher schulformspezifischer Lehrämter differenzielle Vorgehensweisen verfolgen, diversitätssensiblen Unterricht zu gestalten und anzubieten. So weisen die Ergebnisse darauf hin, dass Lehrkräfte, die über eine Lehramtsbefähigung für die Schulform Gymnasium verfügen, die kulturellethnische Heterogenität weniger im Rahmen der Unterrichtsdurchführung und - reflexion berïcksichtigen, sondern vielmehr im Rahmen der Unterrichtsvorbereitung. 
Lehrkräfte mit einer Lehramtsbefähigung für die Primarstufe und die Sekundarstufe I berücksichtigen kulturell-ethnische Vielfalt eher in der direkten Unterrichtsausführung und im Rahmen der anschließenden Bewertung ihres Unterrichts. Da die Lehrkräfte dieser Stichprobe an Gemeinschaftsschulen, demzufolge in ähnlichen schulischen Lernmilieus tätig sind (Holtappels und McElvany 2017), kann dies nur auf die differenzielle Ausbildung im Rahmen des universitären Studiums und des Vorbereitungsdiensts zurückgeführt werden. Für das Alter, welches als weitere Kovariate berücksichtigt wurde und als Indikator für die gesammelte Lehrerfahrung steht, konnte keine Bedeutung für die Gestaltung des Unterrichts festgestellt werden. Dementgegen fanden andere Studien altersspezifische Unterschiede in der Gestaltung des Unterrichts, wobei ältere Lehrkräfte weniger häufig dazu tendieren schülerorientierte und neue Unterrichtskonzepte zu verwenden (z.B. Salvano-Pardieu et al. 2009).

Dies ist möglicherweise mit der Besonderheit der Stichprobe zu erklären, die auch bei der weiteren Interpretation und Übertragbarkeit der Ergebnisse berücksichtig werden muss. Es handelt sich bei den Gemeinschaftsschulen um Modellversuchsschulen; einer zum Zeitpunkt, der Erhebung neugegründete Schulform in Nordrhein-Westfalen (vgl. Holtappels und McElvany 2017). Das mittlere Alter aller Lehrkräfte an Gemeinschaftsschulen in Nordrhein-Westfalen lag zum Zeitpunkt der Erhebung unterhalb dem mittleren Alter aller Lehrkräfte in Nordrhein-Westfahlen, und das Alter der vorliegenden Stichprobe unterhalb des mittleren Alters aller Lehrkräfte an Gemeinschaftsschulen in Nordrhein-Westfalen (vgl. Statistisches Landesamt NRW 2016). Lehrkräfte, die sich entscheiden an eine neugegründete Schulform zu wechseln, die zum Ziel hat, Schule so zu organisieren, dass Schülerinnen und Schüler länger gemeinsam lernen (vgl. Holtappels und McElvany 2017), haben darüber hinaus möglicherweise positivere Einstellungen in Bezug auf das Unterrichten kulturell-ethnisch heterogener Lerngruppen.

Die Ergebnisse sind zudem vor dem Hintergrund der begrenzten Stichprobengröße zu relativieren. Für die Spezifizierung der globalen Modelle war die Fallzahl hinreichend, doch sind die Mehrgruppenmodelle, insbesondere die Substichprobengruppengröße der männlichen Lehrkräfte, grenzwertig. Eine Überprüfung dieser Analysen anhand eines größeren Datensatzes mit weniger spezifischen Lehrkräften könnte zu einer allgemeingültigeren Aussage führen. Darüber hinaus ist auf die hohen Interkorrelationen (vgl. Abb. 1 und 2) zwischen latenten Prädiktoren hinzuweisen. Diese können auf die inhaltliche Nähe der Konstrukte zurückgeführt werden und entsprechen vergleichbaren Befunden anderer Studien (Hartwig und Schwabe 2018).

Gänzlich unbeantwortet muss die Frage bleiben warum bei männlichen und weiblichen Lehrkräfte in der Tendenz differenzielles Zusammenhangsmuster festgestellt werden. Dies kann mit den vorliegenden Daten und den genutzten Methoden nicht beatwortet werden. Diese Fragestellung muss in anschließenden Untersuchungen anhand von beispielsweise qualitativen Methoden untersucht werden. Auch sollten anschließende Forschungen prüfen, ob diversitätssensibler Unterricht heterogenitätsspezifisch differenziell gemessen und erfasst werden kann und, ob heterogenitätsspezifische Einstellungen größere Relevanz dafür zeigen. Auch kann im Rahmen dieser Untersuchung die Frage nicht hinreichend geklärt werden, aus welchen Gründen die 
Einstellungen und Motivation der Lehrkräfte keine Bedeutung für die Unterrichtsvorbereitung hat. Es stellt sich die Frage, ob es andere, in diesem Beitrag nicht berücksichtigte, Faktoren gibt, die für diese Phase bedeutsam sind.

Dennoch leistet die Studie einen Beitrag zum Erkenntnisgewinn im Bereich der Untersuchung überfachlicher Merkmale der professionellen Kompetenz von Lehrkräften und ihre Bedeutung für die Gestaltung eines qualitätsvollen Unterrichts. Dies ist durchaus von Relevanz, da Schülerinnen und Schüler mit Migrationshintergrund durch differenzielle Unterrichtsgestaltung profitieren (Seiz et al. 2016), und weil in einem diversitätssensiblen Unterricht die Wertschätzung einer pluralistischen Gesellschaft im Vordergrund steht (Scheunpflug 2003; Karakaşoğlu 2012). Darüber hinaus ist mit dieser Studie eine sehr differenzierte Form des Unterrichts in den Blick genommen worden. Es zeigt, wie differenziert Lehrkräfte nicht nur ihre eigenen Einstellungen und Motivation wahrnehmen, sondern auch, dass diese differenziellen Wahrnehmungen von spezifischer Bedeutung für die unterschiedlichen Phasen eines differenziellen Unterrichts sind.

Funding Open Access funding provided by Projekt DEAL.

Open Access Dieser Artikel wird unter der Creative Commons Namensnennung 4.0 International Lizenz veröffentlicht, welche die Nutzung, Vervielfältigung, Bearbeitung, Verbreitung und Wiedergabe in jeglichem Medium und Format erlaubt, sofern Sie den/die ursprünglichen Autor(en) und die Quelle ordnungsgemäß nennen, einen Link zur Creative Commons Lizenz beifügen und angeben, ob Änderungen vorgenommen wurden.

Die in diesem Artikel enthaltenen Bilder und sonstiges Drittmaterial unterliegen ebenfalls der genannten Creative Commons Lizenz, sofern sich aus der Abbildungslegende nichts anderes ergibt. Sofern das betreffende Material nicht unter der genannten Creative Commons Lizenz steht und die betreffende Handlung nicht nach gesetzlichen Vorschriften erlaubt ist, ist für die oben aufgeführten Weiterverwendungen des Materials die Einwilligung des jeweiligen Rechteinhabers einzuholen.

Weitere Details zur Lizenz entnehmen Sie bitte der Lizenzinformation auf http://creativecommons.org/ licenses/by/4.0/deed.de.

\section{Literatur}

Ajzen, I. (2001). Nature and operation of attitudes. Annual Review of Psychology, 52(1), 27-58.

Asparouhov, T., \& Muthén, B. (2014). Multiple-group factor analysis alignment. Structural Equation Modeling: A Multidisciplinary Journal, 21(4), 495-508.

$\mathrm{Au}$, K.H. (2009). Isn't culturally responsive instruction just good teaching? Social Education, 73(4), $179-183$.

Bandura, A. (1997). Self-efficacy: the exercise of control. New York: Freeman.

Barber, T. (2002). 'A special duty of care': exploring the narration and experience of teacher caring. British Journal of Sociology of Education, 23(3), 383-395.

Baumert, J., \& Kunter, M. (2006). Stichwort: Professionelle Kompetenz von Lehrkräften. Zeitschrift für Erziehungswissenschaft, 9, 469-520.

Belsley, D. A., Kuh, E., \& Welsch, R.E. (2004). Regression diagnostics: Identifying influential data and sources of collinearity. Hobocken: Wiley \& Sons.

Bem, D. J. (1972). Self-perception theory. Advances in Experimental Social Psychology, 6, 1-62.

Berliner, D.C. (2001). Learning about and learning from expert teachers. International Journal of Educational Research, 35(5), 463-482.

Bernaus, M., Wilson, A., \& Gardner, R. C. (2009). Teachers' motivation, classroom strategy use, students' motivation and second language achievement. Porta linguarum, 12, 25-36. 
Biedermann, H., Brühwiler, C., \& Krattenmacher, S. (2012). Lernangebote in der Lehrerausbildung und Überzeugungen zum Lehren und Lernen. Beziehungsanalysen bei angehenden Lehrpersonen. Zeitschrift für Pädagogik, 58(4), 460-475.

Blömeke, S., Gustafsson, J.E., \& Shavelson, R. J. (2015). Beyond dichotomies. Zeitschrift für Psychologie, 223(1), 3-13.

Blömeke, S., Kaiser, G., \& Lehmann, R. (Hrsg.). (2010). TEDS-M 2008. Professionelle Kompetenz und Lerngelegenheiten angehender Mathematiklehrkräfte für die Sekundarstufe I im internationalen Vergleich. Münster: Waxmann.

Bohner, G., \& Dickel, N. (2011) Attitudes and Attitude Change. Annual Review of Psychology, 62(1), $391-417$

Bress, P. (2000). Gender differences in teaching styles. Forum, 38(4), 26-29.

Brunner, M., Kunter, M., Krauss, S., Baumert, J., Blum, W., Dubberke, T., Jordan, A., Klusmann, U., Tsai, Y., \& Neubrand, M. (2006). Welche Zusammenhänge bestehen zwischen dem fachspezifischen Professionswissen von Mathematiklehrkräften und ihrer Ausbildung sowie beruflichen Fortbildung? Zeitschrift für Erziehungswissenschaft, 9(4), 521-544.

Bundesamt für Migration und Flüchtlinge (2019). Migrationsbericht 2016/2017. http://www.bamf. de/SharedDocs/Anlagen/DE/Publikationen/Migrationsberichte/migrationsbericht-2016-2017.html? nn=1367528. Zugegriffen: 14. Juli 2020.

Byrne, B.M. (2012). Structural equation modeling with Mplus: basic concepts, applications, and programming. New York: Routledge.

Calderhead, J. (1996). Teachers: beliefs and knowledge. In D. C. Berliner \& R. C. Calfee (Hrsg.), Handbook of educational psychology (S. 709-725). New York, NY: Macmillan Library Reference USA, Prentice Hall International.

Cole, A. D. (2006). Scaffolding beginning readers: micro and macro cues teachers use during student oral reading. The Reading Teacher, 59(5), 450-459.

Columna, L., Foley, J. T., \& Lytle, R. K. (2010). Physical education teachers' and teacher candidates' attitudes toward cultural pluralism. Journal of Teaching in Physical Education, 29(3), 295-311.

Cronbach, L.J., \& Snow, R.E. (1977). Aptitudes and instructional methods: a handbook for research on interactions. Oxford: Irvington.

Deci, E.L., \& Ryan, R.M. (1985). The general causality orientations scale: self-determination in personality. Journal of Research in Personality, 19(2), 109-134.

Dickel, N., \& Bohner, G. (2012). Minority and majority influence on attitudes. Psychology: selected papers (S. 249-274).

Docker, J. G., Fraser, B. J., \& Fisher, D. L. (1989). Differences in psychosocial work environment of different types of schools. Journal of Research in Childhood Education, 4, 5-7.

Dubberke, T., Kunter, M., McElvany, N., Brunner, M., \& Baumert, J. (2008). Lerntheoretische Überzeugungen von Mathematiklehrkräften: Einflüsse auf die Unterrichtsgestaltung und den Lernerfolg von Schülerinnen und Schülern. Zeitschrift für pädagogische Psychologie, 22(34), 193-206.

Dweck, C.S., \& Elliot, E. (1988). Goals: An approach to motivation and achievement. Journal of Personality and Social Psychology, 54(1), 5-12.

Eagly, A.H., \& Chaiken, S. (1993). The psychology of attitudes. Fort Worth: Harcourt Brace Jovanovich College Publishers.

Eccles, J. (2011). Gendered educational and occupational choices: Applying the Eccles et al. model of achievement-related choices. International Journal of Behavioral Development, 35(3), 195-201

Eccles, J.S., \& Wigfield, A. (2002). Motivational beliefs, values, and goals. Annual review of psychology, 53(1), 109-132.

Ellemers, N. (2018). Gender stereotypes. Annual Review of Psychology, 69, 275-298.

Ellins, J., \& Porter, J. (2005). Departmental differences in attitudes to special educational needs in the secondary school. British Journal of Special Education, 32, 188-195.

Enders, C. K. (2010). Applied missing data analysis. New York: Guilford.

Evans, C. (2004). Exploring the relationship between cognitive style and teaching style. Educational psychology, 24(4), 509-530.

Fazio, R.H. (2007). Attitudes as object-evaluation associations of varying strength. Social Cognition, 25(5), 603-637.

Fickel, N. (2001). Sequential regression: a neodescriptive approach to multicollinearity (No. 09/2001). EERI Research Paper Series. https://www.econstor.eu/bitstream/10419/142481/1/EERI_RP_2001_ 09.pdf. Zugegriffen: 14. Juli 2020. 
Fischer, N., \& Ehmke, T. (2019). Empirische Erfassung eines „messy constructs“: Überzeugungen angehender Lehrkräfte zu sprachlich-kultureller Heterogenität in Schule und Unterricht. Zeitschrift für Erziehungswissenschaft, 22(2), 411-433.

Fischer, N., Hammer, S., \& Ehmke, T. (2018). Überzeugungen zu Sprache im Fachunterricht: Erhebungsinstrument und Skalendokumentation. In T. Ehmke, S. Hammer, A. Köker, U. Ohm \& B. Koch-Priewe (Hrsg.), Professionelle Kompetenzen angehender Lehrkräfte im Bereich Deutsch als Zweitsprache (S. 149-184). Münster: Waxmann.

Fives, H., \& Buehl, M. M. (2012). Spring cleaning for the "messy" construct of teachers' beliefs: What are they? Which have been examined? What can they tell us? In K. R. Harris, S. Graham, T. Urdan, S. Graham, J.M. Royer \& M. Zeidner (Hrsg.), Individual differences and cultural and contextual factors (APA handbooks in psychology, APA educational psychology handbook, Bd. 2, S. 471-499).

Flake, J. K., Barron, K. E., Hulleman, C., McCoach, B. D., \& Welsh, M.E. (2015). Measuring cost: the forgotten component of expectancy-value theory. Contemporary Educational Psychology, 41, 232-244.

Ford, T. N., \& Quinn, L. (2010). First year teacher education candidates: what are their perceptions about multicultural education. Multicultural Education, 17(4), 18-24.

Frey, A., Taskinen, P., Schütte, K., \& Deutschland, P. K. (Hrsg.). (2009). PISA 2006 Skalenhandbuch: Dokumentation der Erhebungsinstrumente. Münster: Waxmann.

Fuller, A., \& Unwin, L. (2004). Young people as teachers and learners in the workplace: challenging the novice-expert dichotomy. International Journal of Training and Development, 8(1), 32-42.

Furinghetti, F., \& Pehkonen, E. (2002). Rethinking characterizations of beliefs. In G. Leder, E. Pehkonen \& G. Törner (Hrsg.), Beliefs: a hidden variable in mathematics education? (S. 39-57). Dordrecht: Springer.

Gawronski, B., \& Bodenhausen, G. V. (2007). Unraveling the processes underlying evaluation: attitudes from the perspective of the APE model. Social Cognition, 25(5), 687-717.

Gay, G. (2013). Teaching to and through cultural diversity. Curriculum Inquiry, 43(1), 48-70.

Gebauer, M.M., \& McElvany, N. (2013). Einstellungen von Lehramtsanwärtern gegenüber heterogenen Schülergruppen - Skalenhandbuch (Unveröffentlichte Forschungsarbeit). Dortmund: Institut für Schulentwicklungsforschung.

Gebauer, M. M., \& McElvany, N. (2017). Zur Bedeutsamkeit unterrichtsbezogener heterogenitätsspezifischer Einstellungen angehender Lehrkräfte für intendiertes Unterrichtsverhalten. Psychologie in Erziehung und Unterricht, 64(3), 163-180.

Gogolin, I. (2006). Bilingualität und die Bildungssprache der Schule. In P. Mecheril \& T. Quehl (Hrsg.), Die Macht der Sprachen. Englische Perspektiven auf die mehrsprachige Schule (S. 79-85). Münster: Waxmann.

Gomolla, M. (2009). Heterogenität, Unterrichtsqualität und Inklusion. In S. Fürstenau \& M. Gomolla (Hrsg.), Migration und schulischer Wandel: Unterricht (S. 21-43). Wiesbaden: VS.

Grigutsch, S., Raatz, U., \& Törner, G. (1998). Einstellungen gegenüber Mathematik bei Mathematiklehrern. Journal für Mathematik-Didaktik, 19(1), 3-45.

Hall, T. (2002). Differentiated Instruction. Wakefield: National Center on Accessing the General Curriculum. http://www.centerii.org/handbook/Resources/7_B_Differentiating_instruction.pdf. Zugegriffen: 14. Juli 2020.

Hannover, B., \& Kessels, U. (2011). Sind Jungen die neuen Bildungsverlierer? Empirische Evidenz für Geschlechterdisparitäten zuungunsten von Jungen und Erklärungsansätze. Zeitschrift für Pädagogische Psychologie, 25(2), 89-103.

Hartinger, A., Kleickmann, T., \& Hawelka, B. (2006). Der Einfluss von Lehrervorstellungen zum Lernen und Lehren auf die Gestaltung des Unterrichts und auf motivationale Schülervariablen. Zeitschrift für Erziehungswissenschaft, 9(1), 110-126.

Hartmann, P., \& Trautner, H. M. (2009). Die Bedeutung des Pubertätsstatus und des Entwicklungstempos für die Geschlechtsidentität von Mädchen und Jungen in der Adoleszenz. Zeitschrift für Entwicklungspsychologie und pädagogische Psychologie, 41(2), 63-78.

Hartwig, S. J., \& Schwabe, F. (2018). Teacher attitudes and motivation as mediators between teacher training, collaboration, and differentiated instruction. Journal for Educational Research Online, 10(1), $100-122$.

Hartwig, S. J., Schwabe, F., Gebauer, M. M., \& McElvany, N. (2017). Wie beurteilen Lehrkräfte und Lehramtsstudierende Leistungsheterogenität? Ausprägungen, Zusammenhänge und Prädiktoren von Einstellungen und Motivation. Psychologie in Erziehung und Unterricht, 64(2), 94-108.

Hasselhorn, M., \& Grube, D. (2003). Das Arbeitsgedächtnis: Funktionsweise, Entwicklung und Bedeutung für kognitive Leistungsstörungen. Sprache · Stimme · Gehör, 27(01), 31-37. 
Heckhausen, H. (1989). Leistungsmotivation. In H. Heckhausen (Hrsg.), Motivation und Handeln (S. 231-278). Berlin: Springer.

Heckhausen, J., \& Heckhausen, H. (2010). Motivation und Handeln (4. Aufl.). Berlin: Springer.

Henschel, S., Heppt, B., Weirich, S., Edele, A., Schipolowski, S., \& Stanat, P. (2019). Zuwanderungsbezogene Disparitäten. In P. Stanat, S. Schipolowski, N. Mahler, S. Weirich \& S. Henschel (Hrsg.), IQBBildungstrend 2018 (S. 295-332). Münster: Waxmann.

Holtappels, H. G., \& McElvany (2017). Wissenschaftliche Begleitung 2014-2016 zum Schulversuch „Längeres Gemeinsames Lernen - Gemeinschaftsschule“ (Unveröffentlichter Abschlussbericht). Dortmund: Institut für Schulentwicklungsforschung.

Hu, L., \& Bentler, P. M. (1999) Cutoff criteria for fit indexes in covariance structure analysis: Conventional criteria versus new alternatives. Structural Equation Modeling: A Multidisciplinary Journal, 6(1), $1-55$

Huang, S.L. (2007). A comparative study in perceptions of their school environments among secondary science and mathematics teachers. Chinese Journal of Science Education, 15(1), 53-72.

Huang, S. Y.L., \& Fraser, B.J. (2009). Science teachers' perceptions of the school environment: gender differences. Journal of Research in Science Teaching, 46(4), 404-420.

Karakaşoğlu, Y. (2012). Interkulturelle Öffnung von Schulen und Hochschulen. In C. Griese \& H. Marburger (Hrsg.), Interkulturelle Öffnung. Ein Lehrbuch (S. 93-117). München: Oldenbourg.

Klassen, R. M., Al-Dhafri, S., Hannok, W., \& Betts, S. M. (2011). Investigating pre-service teacher motivation across cultures using the Teachers' Ten Statements Test. Teaching and Teacher Education, 27(3), 579-588.

Klieme, E., \& Warwas, J. (2011). Konzepte der individuellen Förderung. Zeitschrift für Pädagogik, 57(6), 805-818.

König, J., Peek, R., \& Blömeke, S. (2008). Zum Erwerb von pädagogischem Wissen in der universitären Ausbildung: Unterscheiden sich Studierende verschiedener Lehrämter und Kohorten? Lehrerbildung auf dem Prüfstand, 1(2), 664-682.

Kooij, D., De Lange, A., Jansen, P., \& Dikkers, J. (2008). Older workers' motivation to continue meanings of age. A conceptual review. Journal of Managerial Psychology, 23, 364-394.

Korneck, F., Krüger, M., \& Szogs, M. (2017). Professionswissen, Lehrerüberzeugungen und Unterrichtsqualität angehender Physiklehrkräfte unterschiedlicher Schulformen. Professionelle Kompetenz von Lehrkräften der Chemie und Physik, 200, 113-133.

Krauss, S., Neubrand, M., Blum, W., Baumert, J., Brunner, M., Kunter, M., \& Jordan, A. (2008). Die Untersuchung des professionellen Wissens deutscher Mathematik-Lehrerinnen und-Lehrer im Rahmen der COACTIV-Studie. Journal für Mathematik-Didaktik, 29(3-4), 233-258.

Kumar, R., Karabenick, S.A., \& Burgoon, J. N. (2015). Teachers' implicit attitudes, explicit beliefs, and the me diating role of respect and cultural responsibility on mastery and performance-focused instructional practices. Journal of Educational Psychology, 107(2), 533.

Kunter, M., \& Voss, T. (2011). Das Modell der Unterrichtsqualität in COACTIV: Eine multikriteriale Analyse. In M. Kunter, J. Baumert \& W. Blum (Hrsg.), Professionelle Kompetenz von Lehrkräften. Ergebnisse des Forschungsprogramms COACTIV (S. 85-113). Münster: Waxmann.

Kyriakides, L., Creemers, B. P., \& Antoniou, P. (2009). Teacher behaviour and student outcomes: suggestions for research on teacher training and professional development. Teaching and Teacher Education, 25(1), 12-23.

Ladson-Billings, G. (2014). Culturally relevant pedagogy 2.0: aka the remix. Harvard Educational Review, 84(1), 74-84.

Lam, Y. H. R., Tse, S. K., Lam, J. W. I., \& Loh, E. K. Y.(2010) Does the gender of the teacher matter in the teaching of reading literacy? Teacher gender and pupil attainment in reading literacy in Hong Kong. Teaching and Teacher Education, 26(4), 754-759

Levy, H. M. (2008). Meeting the needs of all students through differentiated instruction: Helping every child reach and exceed standards. The Clearing House: A Journal of Educational Strategies, Issues and Ideas, 81(4), 161-164.

Lipowsky, F. (2006). Auf den Lehrer kommt es an. Empirische Evidenzen für Zusammenhänge zwischen Lehrerkompetenzen, Lehrerhandeln und dem Lernen der Schüler. In C. Allemann-Ghionda \& E. Terhart (Hrsg.), Kompetenz und Kompetenzentwicklung von Lehrerinnen und Lehrern (Zeitschrift für Pädagogik: Beiheft 51, S. 47-70). Weinheim: Beltz.

Little, R. J. (1988). A test of missing completely at random for multivariate data with missing values. Journal of the American Statistical Association, 83(404), 1198-1202.

Little, T. D., Jorgensen, T. D., Lang, K. M., \& Moore, E. W. G. (2013). On the joys of missing data. Journal of Pediatric Psychology, 39(2), 151-162. 
Maas, C. J., \& Hox, J. J. (2005). Sufficient sample sizes for multilevel modeling. Methodology, 1(3), 86-92. Maio, G. R., \& Haddock, G. (2010). The psychology of attitudes and attitude change. London: SAGE.

Merk, S., Cramer, C., Dai, N., Bohl, T., \& Syring, M. (2018). Faktorielle Validität der Einstellungen von Lehrkräften zu heterogenen Lerngruppen. Journal for Educational Research Online, 10(2), 34-53.

Meschede, N., Fiebranz, A., Möller, K., \& Steffensky, M. (2017). Teachers' professional vision, pedagogical content knowledge and beliefs: On its relation and differences between pre-service and in-service teachers. Teaching and Teacher Education, 66, 158-170.

Muthén, L. K., \& Muthén, B. O. (2018). Mplus User's Guide (8. Aufl.). Los Angeles: Muthén \& Muthén.

Oaksford, L., \& Jones, L. (2001). Differentiated instruction abstract. Tallahassee: Leon County Schools.

Pajares, M.F. (1992). Teachers' beliefs and educational research: cleaning up a messy construct. Review of Educational Research, 62(3), 307-332.

Philipp, R. A. (2007). Mathematics teachers' beliefs and affect. In F. K. Lester (Hrsg.), Second handbook of research on mathematics teaching and learning (S. 257-318). Charlotte, NC: Information Age.

Phinney, J. S., Berry, J. W., Vedder, P., \& Liebkind, K. (2006). The acculturation experience: attitudes, identities and behaviors of immigrant youth. In J. W. Berry, J. S. Phinney, D. L. Sam \& P. Vedder (Hrsg.), Immigrant youth in cultural transition: acculturation, identity, and adaptation across national contexts (S. 71-116). Mahwah: Lawrence Erlbaum.

Reusser, K., \& Pauli, C. (2014). Berufsbezogene Überzeugungen von Lehrerinnen und Lehrern. In E. Terhart, H. Bennewitz \& M. Rothland (Hrsg.), Handbuch der Forschung zum Lehrerberuf (Bd. 2, S. 642-661). Münster: Waxmann.

Rubin, D. B. (1976). Inference and missing data. Biometrika, 63(3), 581-592.

Saborit, J. A. P., Fernández-Río, J., Estrada, J. A. C., Méndez-Giménez, A., \& Alonso, D. M. (2016). Teachers' attitude and perception towards cooperative learning implementation: Influence of continuing training. Teaching and Teacher Education, 59, 438-445.

Salvano-Pardieu, V., Fontaine, R., Bouazzaoui, B., \& Florer, F. (2009). Teachers' sanction in the classroom: effect of age, experience, gender and academic context. Teaching and Teacher Education, 25(1), $1-11$.

Scheunpflug, A. (2003). Stichwort: Globalisierung und Erziehungswissenschaft. Zeitschrift für Erziehungswissenschaft, 6(2), 159-172.

Scheunpflug, A., \& Affolderbach, M. (2019). Bildung im Kontext von Migration und Diversität. In V. Klomann, N. Frieters-Reermann, M. Genenger-Stricker \& N. Sylla (Hrsg.), Forschung im Kontext von Bildung und Migration (S. 11-24). Wiesbaden: Springer VS.

Schmotz, C., \& Blömeke, S. (2009). Zum Verhältnis von fachbezogenem Wissen und epistemologischen Überzeugungen bei angehenden Lehrkräften. Lehrerbildung auf dem Prüfstand, 2(1), 148-165.

Schneider, H. (2009). Nachweis und Behandlung von Multikollinearität. In S. Albers, D. Klapper, U. Konradt, A. Walter \& J. Wolf (Hrsg.), Methodik der empirischen Forschung (S. 221-236). Wiesbaden: Gabler.

Schneider, W., \& Stefanek, J. (2004). Entwicklungsveränderungen allgemeiner kognitiver Fähigkeiten und schulbezogener Fertigkeiten im Kindes-und Jugendalter. Zeitschrift für Entwicklungspsychologie und Pädagogische Psychologie, 36(3), 147-159.

Seiz, J., Decristan, J., Kunter, M., \& Baumert, J. (2016). Differenzielle Effekte von Klassenführung und Unterstützung für Schülerinnen und Schüler mit Migrationshintergrund. Zeitschrift für Pädagogische Psychologie, 30(4), 237-249.

Shulman, L.S. (1986). Those who understand: knowledge growth in teaching. Educational Researcher, 15(2), 4-14.

Snow, R. (1989). Aptitude-treatment interaction as a framework for research on individual differences in learning. In P. Ackerman, R. J. Sternberg \& R. Glaser (Hrsg.), Learning and individual differences (S. 13-59). New York: W.H. Freeman.

Starbuck, G. H. (2003). College teaching styles by gender (Western Social Science Association Annual Meeting, S. 9-12). Las Vegas.

Statistisches Bundesamt (2019). Bevölkerung und Erwerbstätigkeit Bevölkerung mit Migrationshintergrund - Ergebnisse des Mikrozensus 2018. Fachserie 1 Reihe 2.2. https://www.destatis.de/ DE/Themen/Gesellschaft-Umwelt/Bevoelkerung/Migration-Integration/Publikationen/DownloadsMigration/migrationshintergrund-2010220187004.html. Zugegriffen: 14. Juli 2020.

Statistisches Landesamt, N.R.W. (2016). Hauptamtliche und hauptberufliche Lehrkräfte an all gemeinbildenden Schulen in NRW - Schuljahr 2015/2016. https://www.it.nrw/durchschnittsalter-derlehrkraefte-lag-nrw-2015-bei-456-jahren-13050. Zugegriffen: 14. Juli 2020. 
Staub, F. C., \& Stern, E. (2002). The nature of teachers' pedagogical content beliefs matters for students' achievement gains: Quasi-experimental evidence from elementary mathematics. Journal of educational psychology, 94(2), 344.

Stocké, V. (2004). Entstehungsbedingungen von Antwortverzerrungen durch soziale Erwünschtheit: Ein Vergleich der Prognosen der rational-choice Theorie und des Modells der frame-Selektion. Zeitschrift für Soziologie, 33(4), 303.

Strack, F., \& Deutsch, R. (2004). Reflective and impulsive determinants of social behavior. Personality and Social Psychology Review, 3, 220-247.

Tharp, R.G. (1989). Psychocultural variables and constants: Effects on teaching and learning in schools. American Psychologist, 44(2), 349-359. https://doi.org/10.1037/0003-066X.44.2.349

Thorndike, R. L. (1982). Applied psychometrics. Boston: Houghton Mifflin.

Tillmann, K. J. (2008). Viel Selektion - wenig Leistung: Erfolg und Scheitern in deutschen Schulen. In R. Lehberger \& U. Sandfuchs (Hrsg.), Schüler fallen auf. Heterogene Lerngruppen in Schule und Unterricht (S. 62-78). Bad Heilbrunn: Klinkhardt.

Tomlinson, C. A. (2014). The differentiated classroom: responding to the needs of all learners. Alexandria: ASCD.

Tomlinson, F., \& Egan, S. (2002). Organizational sensemaking in a culturally diverse setting: limits to thevaluing diversity'discourse. Management Learning, 33(1), 79-97.

Trautner, H. M. (1991). Theorien und Befunde (2. Aufl.). Göttingen: Hogrefe.

Tricarico, K., \& Yendol-Hoppey, D. (2012). Teacher learning through self-regulation: an exploratory study of alternatively prepared teachers' ability to plan differentiated instruction in an urban elementary school. Teacher Education Quarterly, 39(1), 139-158.

Tschannen-Moran, M., Hoy, A.W., \& Hoy, W. K. (1998). Teacher efficacy: Its meaning and measure. Review of Educational Research, 68(2), 202-248.

Voss, T., Kleickmann, T., Kunter, M., \& Hachfeld, A. (2013). Mathematics teachers' beliefs. In M. Kunter, J. Baumert, W. Blum, U. Klusmann, S. Krauss \& M. Neubrand (Hrsg.), Cognitive activation in the mathematics classroom and professional competence of teachers (S. 249-271). Boston: Springer.

Voss, T., Kunter, M., Seiz, J., Hoehne, V., \& Baumert, J. (2014). Die Bedeutung des pädagogisch-psychologischen Wissens von angehenden Lehrkräften für die Unterrichtsqualität. Zeitschrift für Pädagogik, 60(2), 184-201.

Watermann, R., \& Baumert, J. (2006). Entwicklung eines Strukturmodells zum Zusammenhang zwischen sozialer Herkunft und fachlichen und überfachlichen Kompetenzen: Befunde national und international vergleichender Analysen. In J. Baumert, P. Stanat \& R. Watermann (Hrsg.), Herkunftsbedingte Disparitäten im Bildungswesen: Differenzielle Bildungsprozesse und Probleme der Verteilungsgerechtigkeit (S. 61-94). Wiesbaden: VS.

Wiater, W. (2012). Kulturdifferenz verstehen - Bedingungen - Möglichkeiten - Grenzen. In W. Wiater \& D. Manschke (Hrsg.), Verstehen und Kultur Mentale Modelle und kulturelle Prägungen (S. 15-30). Wiesbaden: VS.

Wigfield, A., Eccles, J.S., \& Pintrich, P.R. (1996). Development between the ages of 11 and 25. In D. C. Berliner \& R. C. Calfee (Hrsg.), Handbook of educational psychology (S. 148-185). New York: Macmillan.

Wyss, C. (2013). Unterricht und Reflexion. Eine mehrperspektivische Untersuchung der Unterrichts- und Reflexionskompetenz von Lehrkräften (Empirische Erziehungswissenschaft, Band 44). Münster: Waxmann.

Zemore, S. E., Fiske, S. T., \& Kim, H. J. (2012). Gender stereotypes and the dynamics of social interaction. In T. Eckes \& H. M. Trautner (Hrsg.), The developmental social psychology of gender (S. 221-256). New York: Psychology Press. 